\title{
Tecnura
}

\section{Desempeño ambiental de recintos habitacionales. Comparación de simulaciones, monitorización y percepción de residentes en seis viviendas de Concepción, Chile}

\author{
Environmental Performance of Residential Rooms. \\ Comparison between Simulations, Monitoring and Perception \\ of Dwellers in Six Houses of Concepcion, Chile \\ Rodrigo García Alvarado1, Rodrigo Herrera Ojeda², Cristián Muñoz Viveros³ \\ Gerth Wandersleben ${ }^{4}$
}

Fecha de recepción: 9 de octubre de 2014

Fecha de aceptación: 18 de septiembre de 2015

Cómo citar: García Alvarado, R., Herrera Ojeda, R., Muñoz Viveros, C., \& Wandersleben, G. (2016). Desempeño ambiental de recintos habitacionales. Comparación de simulaciones, monitorización y percepción de residentes en seis viviendas de concepción, Chile. Revista Tecnura, 20(47), 71-84. doi: 10.14483/udistrital.jour. tecnura.2016.1.a06

\section{Resumen}

Los estudios y proyectos habitacionales consideran las viviendas de manera unitaria, sin diferenciar las condiciones ambientales por recintos, aunque en la vivencia cotidiana se reconocen variaciones. Este artículo realiza una comparación entre los datos proporcionados por simulaciones computacionales de desempeño térmico y monitoreos físicos de recintos, que registran valores existentes, y percepciones de sus habitantes en torno a su ambiente residencial en seis viviendas de Concepción, Chile. Con el fin de caracterizar las diferencias ambientales que surgen en los hogares, como también las originadas en los distintos recintos, en lo que es la construcción de la confortabilidad. Las simulaciones expresan algunas diferencias relevantes entre recintos (demandas energéticas anuales de $12 \mathrm{~kW} \mathrm{~h} / \mathrm{m}^{2}$ hasta 330 $\mathrm{kW} \mathrm{h} / \mathrm{m}^{2}$ ), mientras las mediciones físicas revelan valores más acotados (diferencias entre temperaturas ambientales de menos de $5^{\circ} \mathrm{C}$ ). Las entrevistas y observación de residentes sugieren apreciaciones similares, pero a la vez aportan otros aspectos (como una mayor valoración de los niveles superiores y habitaciones con mayor luminosidad). Los resultados y conclusiones emanados del estudio aportan datos que pueden resultar de interés comparativo para la planificación y diseño habitacional en la zona, pero también un análisis que integra las distintas dimensiones trabajadas del confort residencial y sus variaciones entre los recintos habitacionales, lo que resulta incidente para el ámbito de las políticas y acciones de mejoramiento ambiental de las viviendas, destinadas a aumentar la calidad residencial.

Palabras clave: Ambiente interior, Chile, confortabilidad ambiental, vivienda

Arquitecto, doctor en Arquitectura, departamento de Diseño y Teoría de Arquitectura Universidad del Bío-Bío. Concepción, Chile. Contacto: rgarcia@ubiobio.cl

Antropólogo, doctor en Antropología Social y Cultural, académico Carrera Antropología Universidad de Concepción. Concepción, Chile. Contacto: rherrerao@udec.cl

3 Arquitecto, magíster en Hábitat Sustentable y Eficiencia Energética, docente de la Escuela de Arquitectura e Investigador del Centro de Investigación en Tecnologías de la Construcción CITEC Universidad del Bío-Bío. Concepción, Chile. Contacto: chilecmunoz@ubiobio.cl

Arquitecto, docente de la Escuela de Arquitectura Universidad del Bío-Bío. Concepción, Chile. Contacto: gerthwwm@ubiobio.cl 


\begin{abstract}
The housing studies and design regards homes like one indoor environment, without recognizing differences between rooms, although in the daily living it perceives variations. This article presents a comparison between data from computer simulations of thermal performance and physical monitoring that records existing values of rooms, with perceptions of its inhabitants around their residential environment, from six houses located in Concepción, Chile. In order to characterize environmental differences that arise in homes, as well as those originated in different rooms, which represents comfort building. Simulations express some significant differences by venues (anual energy demands from $12 \mathrm{kWh} / \mathrm{m} 2$ to
\end{abstract}

$330 \mathrm{kWh} / \mathrm{m} 2$ ), while physical measurements reveal more limited values (differences between environmental temperatures less than $5^{\circ} \mathrm{C}$ ). Interviews and observation of residents suggest nearby considerations, but also provide other aspects (like higher value of upper story and rooms with high luminance). The outcomes and conclusions of the study provide data that can be of comparative interest for housing planning and design, but also an analysis that integrates the diverse studied dimensions, which is incident to environmental policies and actions improving housing, targeted to increase the residential quality.

Keywords: Chile, comfort, energy simulation, environment, housing, indoor.

\section{INTRODUCCIÓN}

El creciente consumo energético residencial está motivando estudios ambientales de las viviendas para reducir la demanda de combustibles y mejorar las condiciones de clima interior, especialmente en cuanto a los niveles de confort térmico. Estos análisis utilizan con frecuencia simulaciones térmicas de los edificios (Krygiel y Nies, 2008; Hensen y Lambert, 2011; Jankovic, 2012; Anderson, 2014), que consideran resultados del total del ambiente interior, sin diferenciar los distintos recintos, en especial en los edificios de tamaño pequeño, como las viviendas unifamiliares, que poseen varias habitaciones, simplificándolas en modelos unizona (Hendron y Engebretch, 2010). Sin embargo, cuando se realizan mediciones de variables ambientales, como temperatura, humedad, iluminación o calidad del aire, suelen evidenciarse diferencias entre habitaciones (Sarmiento y Hormazábal, 2003; León, Muñoz, León y Bustamante, 2011; Beizaee, Lomas y Firt, 2013). También las consultas sobre confort térmico a los usuarios indican variaciones de percepción relativas al interior de las viviendas (Peeters, Dear, Hensen y D'Haeseleer, 2009), y la experiencia cotidiana revela cambios en la situación térmica o lumínica entre recintos (Smith, 2012), en particular en viviendas aisladas y localizadas en climas de mayor oscilación térmica. También, Chiou (2009) registró que el control diferenciado de los espacios interiores puede permitir sustanciales ahorros energéticos.

En Chile, se han realizado varias revisiones ambientales en viviendas (como los estudios de Ambiente Consultores, 2007; Bustamante, 2009; Centro de Desarrollo Tecnológico, 2010; Fissore, 2012) que revelan condiciones interiores deficientes $y$ altas demandas energéticas, según las distintas zonas climáticas del país, aunque sin diferenciar resultados acerca de los recintos interiores de las unidades habitacionales.

Por ejemplo, para viviendas unifamiliares en la ciudad de Concepción, principal centro urbano del sur del país, los estudios previamente mencionados indican requerimientos térmicos sobre los $100 \mathrm{~kW} \mathrm{~h} / \mathrm{m}^{2}$, principalmente debidos a calefacción en invierno.

Esta ciudad se encuentra localizada en los $36^{\circ} 46^{\prime} 22^{\prime \prime}$ de latitud sur y $73^{\circ} 03^{\prime} 47^{\prime \prime}$ de longitud oeste, y posee un clima templado-húmedo (figura 1), con estaciones marcadas con temperaturas de $11{ }^{\circ} \mathrm{C}$ a $22{ }^{\circ} \mathrm{C}$ en verano, y de $6{ }^{\circ} \mathrm{C}$ a $13{ }^{\circ} \mathrm{C}$ en 
invierno, $70 \%$ de humedad relativa, precipitaciones cercanas a los $1.110 \mathrm{~mm}$ anuales e irradiación global media diaria de 1,8 a 6,6 kW h/m². El área urbana de Concepción aloja aproximadamente un millón de habitantes, en unas 250.000 viviendas, que en un $85 \%$ son aisladas o adosadas, en uno o dos pisos, combinando albañilería y estructura de madera (Cámara Chilena de la Construcción, 2011). Desde el año 2000 se consideran en el país reglamentos de transmitancia térmica para la techumbre, y desde 2007 para la envolvente general de las viviendas, y actualmente se aplican programas de rehabilitación y calificación energética. Sin embargo, estos consideran un análisis global de la edificación, y un confort interior homogéneo que escasamente se alcanza.

Las condiciones de confort térmico son determinadas habitualmente según un estándar similar en todas las zonas climáticas, tipologías constructivas y recintos (Fanger, 1973); a partir del cual se determinan los requerimientos energéticos de los edificios. Sin embargo, una caracterización por recinto permitiría focalizar los esfuerzos de reacondicionamiento constructivo en aquellas habitaciones donde se pueda generar mayor reducción sobre la demanda de energía. También, el modelo estándar de confort global ha sido discutido reconociendo las diferencias culturales y geográficas de la población (Gómez-Aspeitía, Bojórquez y Ruiz, 2007). Igualmente se han advertido condiciones del propio edificio y de la ocupación de los espacios que influyen en la sensación ambiental (Elzeyadi, 2009; Frontczak, 2011), pero no han sido cabalmente definidos. En este artículo se pretende caracterizar las diferencias interiores ambientales entre habitaciones en viviendas unifamiliares a través de la comparación de simulaciones, monitoreos y percepción de residentes en seis casos localizados en Concepción, Chile. En la primera parte del texto se describe el registro realizado y sus resultados generales; en la segunda parte, de acuerdo con los valores obtenidos se analizan características arquitectónicas de los recintos, para identificar condiciones de los recintos que permitan orientar los estudios y acciones de mejoramiento ambiental en las viviendas.

\section{METODOLOGÍA}

Se privilegió el uso simultáneo de técnicas que recopilaran información de carácter cuantitativo y cualitativo, para después generar resultados

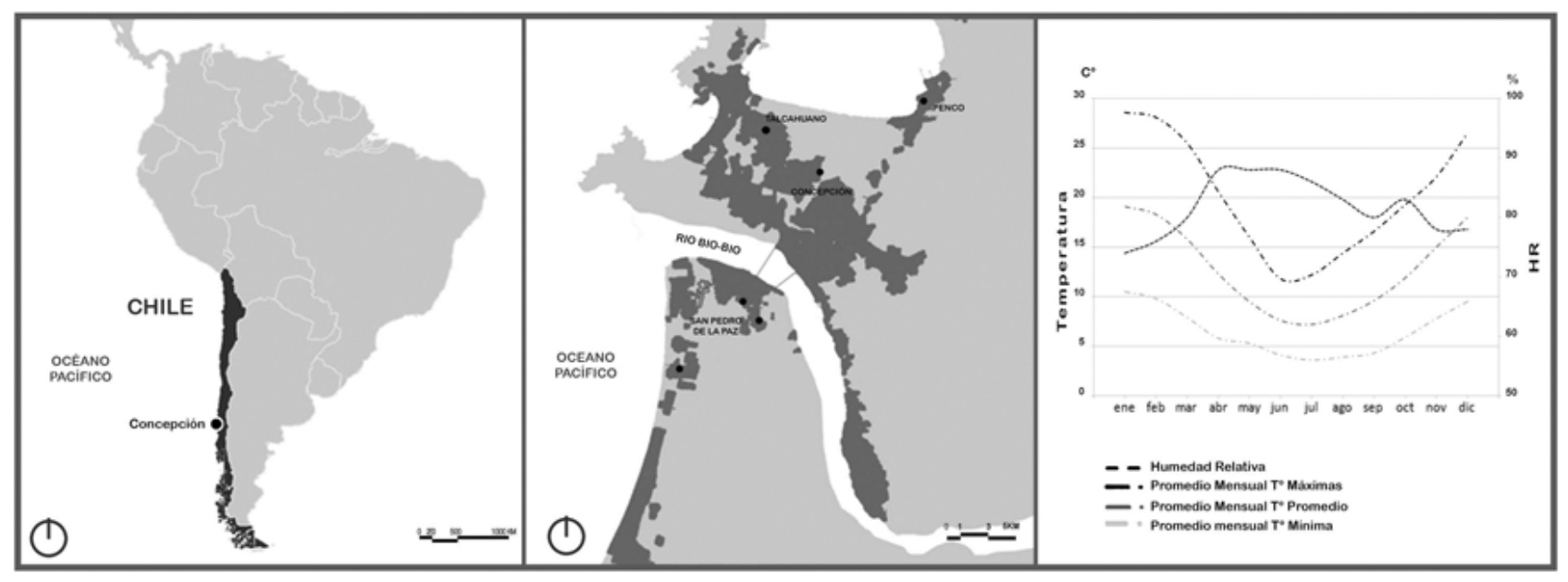

Figura 1. Localización, área urbana (de los casos) y clima de Concepción

Fuente: elaboración propia. 
a través del cruce de los datos indagados. En el caso de la aplicación de simulaciones computacionales, el objetivo era proyectar escenarios de desempeño térmico de las viviendas, revisando diferencias entre los diferentes recintos que las componen. Los monitoreos físicos tenían por objetivo buscar valores reales de comportamiento en las distintas viviendas y habitaciones, en periodos específicos. Por último, las visitas intensivas buscaban dar cuenta de los usos y percepciones que los respectivos ocupantes otorgaban al hogar y a sus diferentes ambientes. El cruce de la información recopilada a través de estas tres técnicas permitió un análisis integral que permitió llegar a características de los recintos que permiten aportar al mejoramiento de las viviendas, integrando la perspectiva de su ocupación, su materialidad y las demandas energéticas requeridas.

Las viviendas analizadas corresponden a la tipología habitacional unifamiliar, predominante en distintas ubicaciones del área metropolitana de Concepción (figura 2), que alojan a más del 70 $\%$ de la población (cerca de 600.000 habitantes). Se seleccionaron viviendas mayores a la media de superficie registrada en la zona $\left(60 \mathrm{~m}^{2}\right)$ para revisar ejemplos con variedad de recintos que además ilustran el desarrollo inmobiliario local y que generan los más altos consumos energéticos. Los casos estudiados fueron elegidos de una muestra convocada entre estudiantes universitarios, quienes revisaron sus propias residencias o de parientes, quienes se seleccionaron según la diversidad de su estructura familiar y disponibilidad de los usuarios a participar en el ejercicio. En cada vivienda se realizó primero una recopilación de características constructivas, equipamiento y ocupación. Luego, se elaboraron simulaciones computacionales de desempeño térmico, con una modelación zonificada por recintos, efectuando un cálculo dinámico (hora a hora) que permitió obtener resultados de demanda energética mensuales y anuales según un nivel de confort térmico dado. En las monitorizaciones físicas se registraron valores de calidad del aire, iluminación, temperatura y humedad relativa, exterior e interior, en cuatro habitaciones (con sensores superficiales y de aire), durante una semana de verano y otra de invierno. Además, se realizaron ensayos y determinaciones

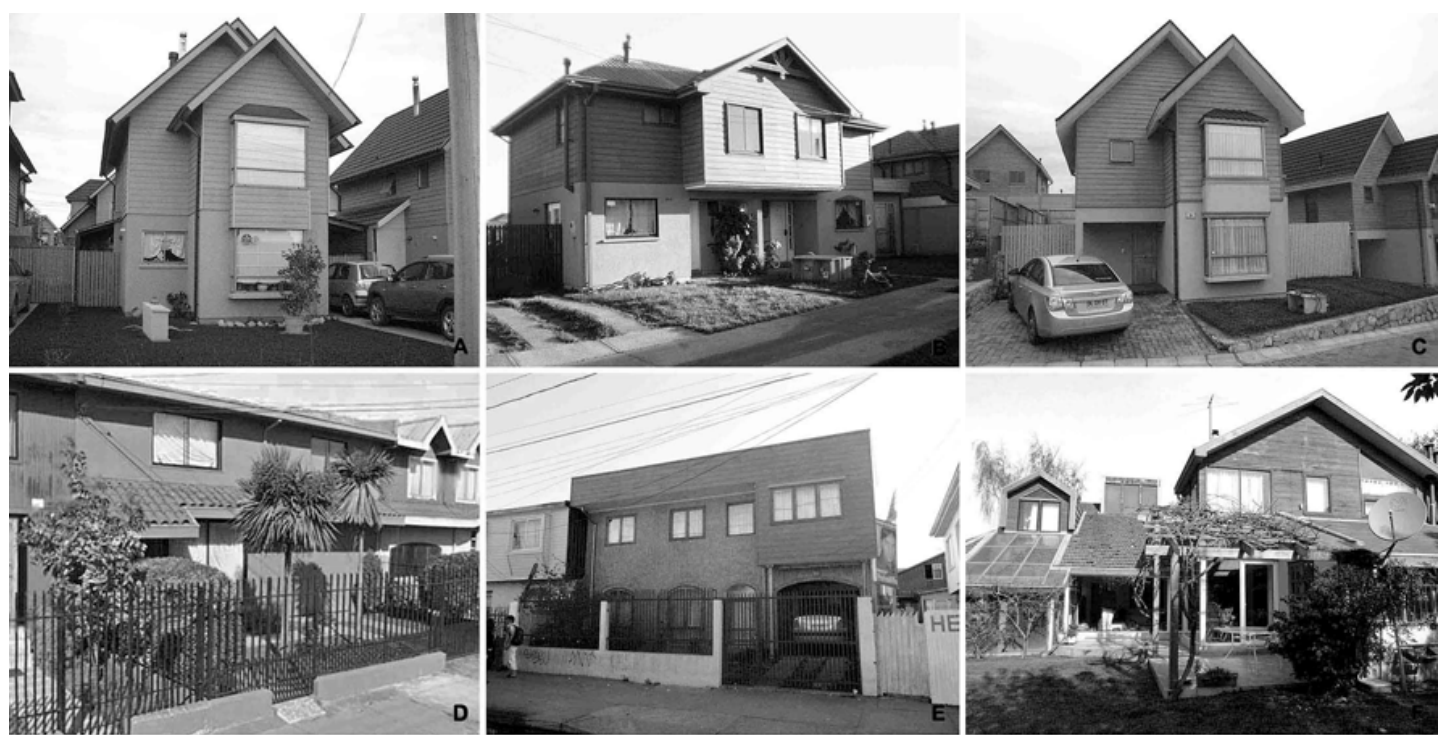

Figura 2. Fotos de las viviendas estudiadas

Fuente: elaboración propia 
de características físicas y comportamiento de la envolvente in situ de termoflujometrías, termografías, mediciones de infiltración de aire y consumo eléctrico por demanda de calefacción. En las consultas se efectuaron encuestas de percepción térmica normalizadas, entrevistas y visitas de un día completo de duración en dos periodos, con observaciones de las actividades y preguntas abiertas sobre experiencias y preferencias de uso de los espacios a todos los adultos de cada vivienda. Estas revisiones fueron realizadas por distintos profesionales, elaborando informes resumidos y analizados por el grupo de investigadores.

De los seis casos revisados (tabla 1), cinco son viviendas de dos pisos y una de tres, en volúmenes independientes, parcialmente adjuntas a construcciones vecinas y emplazadas en la parte central de sitios rectangulares de zonas residenciales. Todas están habitadas por un grupo familiar permanente, y edificadas con un primer piso de albañilería confinada (con valores de transmitancia térmica $U$ ) de 1,7 a $2,4 \mathrm{~W} / \mathrm{m}^{2}{ }^{\circ} \mathrm{C}$ ) y pisos superiores de entramado de madera con aislación (con valores $U$ de 0,8 a $1,4 \mathrm{~W} / \mathrm{m}^{2}{ }^{\circ} \mathrm{C}$ ). Tres de las viviendas son recientes (con menos de tres años de construcción), y ocupadas por familias jóvenes con niños pequeños. Las otras tres fueron construidas hace varias décadas, poseen mayor dimensión por ampliaciones y alojan residentes de más edad. Todas las viviendas presentan una organización espacial similar: en el primer piso se localizan uno o más recintos de estar y comedor, habitualmente integrados (sin muros divisorios), con circulaciones laterales y escalera, además de cocina y algunos dormitorios y baños separados; y en el segundo piso se presentan varios dormitorios y baños en torno a un vestíbulo. Los recintos son ortogonales y de tamaños similares (excepto baños y pasillos más reducidos), dispuestos en una configuración rectangular de bordes variados (figura 3).

\section{Simulaciones}

Se realizaron simulaciones de comportamiento térmico de los seis casos, con el software Autodesk Ecotect Analysis, y con DesignBuilder (usando EnergyPlus como motor de cálculo). Para analizar las diferencias interiores se definieron zonas por recintos, comparando resultados obtenidos con Ecotect ya que es más consistente en la definición de zonas. En Ecotect los recintos se definen por volúmenes independientes que comparten muros o pisos, mientras que en DesignBuilder se subdivide un volumen global, lo que limita la modificación y análisis de zonas. En los dos programas se indica la configuración de vanos (puertas y ventanas), y propiedades termofísicas de los materiales (densidad, conductividad y calor específico), además

Tabla 1. Casos analizados

\begin{tabular}{|c|c|c|c|c|c|c|}
\hline & A & B & C & D & $\mathbf{E}$ & $\mathbf{F}$ \\
\hline Superficie total $\left(\mathrm{m}^{2}\right)$ & 79,47 & 91,97 & 118,8 & 135,60 & 170,00 & 207,16 \\
\hline Año de ejecución & 2011 & 2010 & 2010 & 1957 & 1940 & 1988 \\
\hline Cantidad de niveles & 2 & 3 & 2 & 2 & 2 & 2 \\
\hline Cantidad de ocupantes & $\begin{array}{l}2 \text { adultos }+ \\
2 \text { niños }\end{array}$ & $\begin{array}{l}2 \text { adultos }+ \\
2 \text { niños }\end{array}$ & $\begin{array}{l}2 \text { adultos }+ \\
2 \text { niños }\end{array}$ & $\begin{array}{c}2 \text { ad. may. }+ \\
3 \text { adultos }\end{array}$ & 4 adultos & $\begin{array}{c}2 \text { adultos } \\
\text { mayores }\end{array}$ \\
\hline Cantidad de zonas & 12 & 14 & 17 & 10 & 15 & 23 \\
\hline $\begin{array}{l}\text { Gasto en calefacción } \\
\text { (kW h/año) }\end{array}$ & 8.205 & 6.368 & 7.338 & 4.418 & 5.511 & 18.243 \\
\hline $\begin{array}{l}\text { Demanda de Calefac- } \\
\text { ción (kW h/año) }\end{array}$ & 5.883 & 6.115 & 7.806 & 18.113 & 8.283 & 17.311 \\
\hline Por m² (kW h/año) & 74 & 66 & 66 & 134 & 49 & 80 \\
\hline
\end{tabular}

Fuente: elaboración propia 

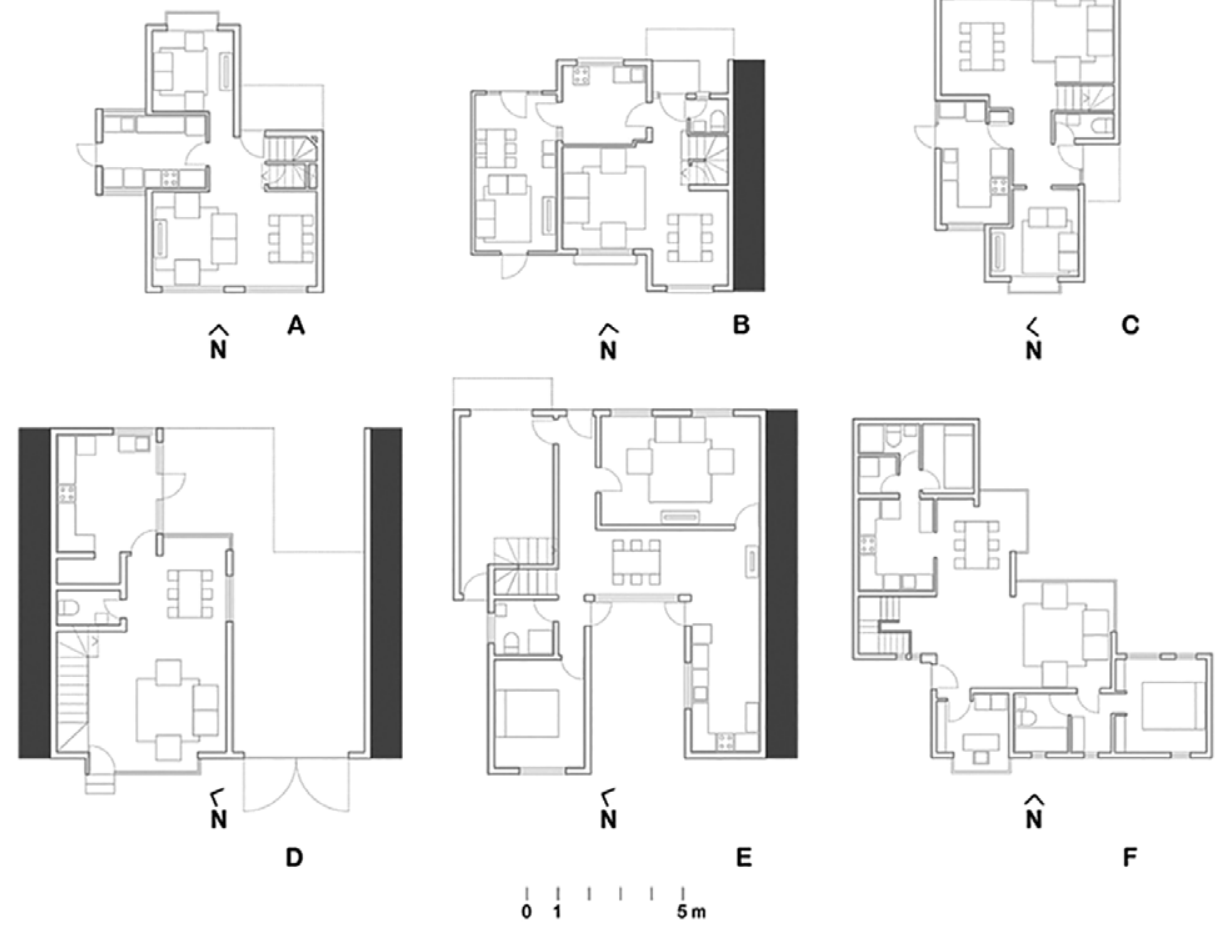

Figura 3. Plantas de las viviendas

Fuente: elaboración propia

del espesor y detalle constructivo, para calcular la transmitancia térmica (valor $U$ ). Además, se indicaron las funciones y calendarios de ocupación por recinto, ganancias internas, equipos de calefacción y eficiencia. Se estableció una renovación de aire similar para todos los casos equivalente a $1,5 \mathrm{ACH}$, correspondiente a la media de esta tipología en la zona (Figueroa et al., 2013). Se estableció una base climática según la localización (IWEC), orientación solar y condiciones generales del entorno. Por último, se definió una banda de confort de $18{ }^{\circ} \mathrm{C}$ a $25^{\circ} \mathrm{C}$ según cálculo basado en el confort adaptativo para la localidad en estudio (basado en ASHRAE 55).

\section{Monitorizaciones}

A través de las mediciones físicas se busca obtener valores del comportamiento de los elementos constructivos y las condiciones ambientales en periodos específicos, mediante el uso de instrumental (tabla 2). Para el monitoreo de temperaturas (del aire y superficiales), humedad relativa del aire, concentración de $\mathrm{CO}_{2}$ e iluminación, se seleccionó el instrumento HOBO U26-001 (registrador de oxígeno disuelto), por su confiabilidad y capacidad para recoger datos en espacios habitados. En termoflujometría (flujos de calor por paramentos verticales exteriores) se recurrió a un equipo compuesto por termocuplas y un microvoltímetro, con el método definido por la norma ASTM C 1046. Para las imágenes infrarrojas de valor térmico de superficies, o termografías, se utilizó una cámara termográfica Fluke y el procedimiento de mediciones se hizo según la norma ISO 6781:1883 modificada. La magnitud de infiltración de aire en las viviendas se midió por presurización con ventilador ubicado en las puertas de acceso (test blowerdoor), según norma UNE13728:2003. 
Tabla 2. Mediciones realizadas

\begin{tabular}{|c|c|c|}
\hline $\begin{array}{l}\text { Exigencia/ } \\
\text { Desempeño }\end{array}$ & Parámetros/Indicador & Técnica /Procedimiento \\
\hline \multirow{3}{*}{$\begin{array}{l}\text { Demanda energética } \\
\text { Aislación térmica } \\
\text { Hermeticidad }\end{array}$} & Energía, E (kWh) & $\begin{array}{l}\text { Monitorización eléctrica según protocolo definido con equipo } \\
\text { HOBO Data-Logger UX120-017 entradas de pulsos } 512 \text { kb de } \\
\text { memoria }\end{array}$ \\
\hline & $\begin{array}{l}\text { Transmitancia Térmica, } \\
\mathrm{U}\left(\mathrm{W} / \mathrm{m}^{2}{ }^{\circ} \mathrm{C}\right)\end{array}$ & Determinación de termoflujometría in situ según ASTM C 1046 \\
\hline & $\begin{array}{l}\text { Cambios de aire }(\mathrm{ACH}) \\
\text { a } 50 \mathrm{~Pa}(1 / \mathrm{h})\end{array}$ & $\begin{array}{l}\text { Determinación de hermeticidad mediante presurización por medio } \\
\text { de ventilador (test blower door) según UNE13728:2003 }\end{array}$ \\
\hline Calidad del aire & $\begin{array}{l}\text { Concentración de } \mathrm{CO}_{2} \\
\mathrm{C}(\mathrm{ppm})\end{array}$ & $\begin{array}{l}\text { Monitorización de concentraciones de } \mathrm{CO}_{2} \text { según protocolo con } \\
\text { Equipo } \mathrm{HOBO} \cup \mathrm{NI}-\mathrm{K} 30\end{array}$ \\
\hline \multirow{4}{*}{ Confort higrotérmico } & Temperatura del aire, $\mathrm{Ta}\left({ }^{\circ} \mathrm{C}\right)$ & $\begin{array}{l}\text { Monitorización higrotérmica según protocolo definido con: } \\
\text { Equipo HOBO Data Logger U12-013, } 2 \text { salidas análogas Ta/HR }\end{array}$ \\
\hline & $\begin{array}{l}\text { Temperatura superficial exte- } \\
\text { rior, Tse }\left({ }^{\circ} \mathrm{C}\right)\end{array}$ & $\begin{array}{l}\text { Equipo HOBO Data Logger TMC6-HE: Sensorde Temperatura de } \\
\text { pared o tubería }\end{array}$ \\
\hline & $\begin{array}{l}\text { Temperatura superficial inte- } \\
\text { rior, Tsi }\left({ }^{\circ} \mathrm{C}\right)\end{array}$ & $\begin{array}{l}\text { Equipo HOBO Data Logger TMC6-HE: sensor de temperatura de } \\
\text { pared o tubería }\end{array}$ \\
\hline & $\begin{array}{l}\text { Humedad relativa del aire, } \\
\text { HR }(\%)\end{array}$ & $\begin{array}{l}\text { Monitorización higrotérmica según protocolo definido con: equipo } \\
\text { HOBO Data Logger U12-013, } 2 \text { salidas análogas Ta/HR }\end{array}$ \\
\hline Confort lumínico & $\begin{array}{l}\text { Niveles de iluminancia, } \\
\text { I ( Lux) }\end{array}$ & $\begin{array}{l}\text { Monitorización de iluminancia según protocolo definido con equipo } \\
\text { HOBO Data Logger U12-012 }\end{array}$ \\
\hline $\begin{array}{l}\text { Termografía. Condición } \\
\text { térmica de la superficie. } \\
\text { Emisiones infrarrojas. } \\
\text { Detección de puentes } \\
\text { térmicos. }\end{array}$ & $\begin{array}{l}\text { Temperatura superficial } \\
\text { interior y exterior a través de } \\
\text { imagen infrarroja }\end{array}$ & $\begin{array}{l}\text { Cámara Fluke, modelo Ti25. Técnica y protocolo desarrollado por } \\
\text { Citec en base a norma ISO 6781:1883 modificada. }\end{array}$ \\
\hline
\end{tabular}

Fuente: elaboración propia

Para implementar el procedimiento de instalación de equipos, tiempos de medición y calibración de equipos, se definió, en una primera instancia, un protocolo de trabajo (experimentado en la vivienda de uno de los investigadores), que permitió programar mediciones de verano entre diciembre de 2012 y enero de 2013, y posteriormente de invierno, entre julio y agosto de 2013. El trabajo se orientó a estudiar relaciones de interés entre las variables físicas del desempeño energético y ambiental de las viviendas (temperaturas, humedad y calidad del aire interior principalmente), las variables constructivas propias del diseño (materialidad, aislación e inercia térmica, orientación y ubicación de los recintos, agrupamiento, etc.) y las condiciones de ocupación. Los datos recopilados en cada periodo fueron extraídos de los sensores y traspasados a gráficos y tablas para su evaluación.

\section{Visitas}

Para analizar la percepción de los residentes se utilizaron distintos procedimientos. Primero, una consulta para un registro global de información relevante a los estudiantes relacionados con los casos. Luego se realizó una encuesta de satisfacción térmica según método Fanger (Fanger, 1973) que reconoce la norma de confort ISO 7730. Posteriormente se efectuaron visitas focalizadas, siguiendo una adaptación de la técnica cualitativa del shadowing (Jirón, 2011). Este procedimiento implicó acompañar a los residentes adultos durante un día, observando y registrando sus actividades 
domésticas, en diálogo con ellos para conocer la vivencia corporal, tanto como las expectativas implicadas en la construcción simbólica de los espacios domésticos. El estudio pretendía dar cuenta del tiempo y lugar de las actividades cotidianas, pero también ayudar a resolver interrogantes como: ¿Tendrán significados específicos los dormitorios, la cocina, el comedor? O, ¿cómo se construyen y exteriorizan las valoraciones otorgadas a los distintos recintos dentro del hogar? Ello, asumiendo que la confortabilidad ambiental se escenifica por medio de la determinación de patrones de uso cotidiano de los distintos recintos, y su funcionalización orientada para acoger diferentes formas de apropiación. Este proceso consideró asumir la técnica en su doble intencionalidad: como actividad/metodología que fortalecía un acercamiento visual; $y$, por otra, apelando a un nivel discursivo al pedirle a los residentes que detallaran el uso de los espacios, los significados y condiciones de cada uno (Jirón, 2011). La apropiación física y simbólica de los espacios domésticos surge así en su versión concreta del uso, pero también en los discursos elaborados que los cualifican y ubican como partes de un conjunto mayor.

La encuesta según el método Fanger se aplicó en diciembre de 2012 a dos ocupantes adultos de cada una de las viviendas, en horario de mediodía con una temperatura media de $22,62{ }^{\circ} \mathrm{C}$ con $60,7 \%$ de humedad relativa, es decir dentro del rango de confort considerado. Se les entrevistó en el recinto de estar, en los que los encuestados portaban ropa ligera, entregando para todos los casos un resultado de satisfacción térmica (voto medio estimado -PMV- = 0 y personas previstas en disconfort $-\mathrm{PMD}-=0,5$ ). Sin embargo, en las respuestas se advierten tendencias diferentes en los hombres respecto a las mujeres, y las personas mayores revelan sentirse más satisfechas. En las entrevistas expresaron también condiciones de sensación térmica distintas según sectores de la casa y estaciones del año, sobre todo en relación a los niveles de la casa y por el uso de calefacción en invierno, estableciéndose que no lograban una satisfacción completa.
Las visitas se realizaron entre abril y junio de 2013, con una recopilación general de actividades y percepciones domésticas. Y luego, en agosto de 2013 se utilizó una pauta más específica de consultas sobre recintos e intereses, para lo cual se indagó sobre la historia familiar; apreciaciones de viviendas anteriores utilizadas; del entorno; comportamiento ambiental, y expectativas futuras.

\section{RESULTADOS}

Las simulaciones, monitoreos y estudios de percepción en las viviendas estudiadas presentan algunos resultados coincidentes por recintos, aspectos que podrían ser relevantes en el diseño residencial.

Las visitas advirtieron una situación semejante en todas las viviendas, con rutinas de ocupación definidas por la edad, condición laboral y rol dentro del grupo familiar, con variaciones ocasionales y modificaciones de residentes entre un año y otro, lo que hablaría de una movilidad interna a considerar. El hallazgo más relevante es que, a despecho de las condiciones térmicas o ambientales, la comodidad se advierte ligada a la independencia espacial; esto es, el aislamiento físico, auditivo y visual. Así, se reconoce la importancia de una privacidad colectiva, pero más aún, la de una individual. Por su parte, las condiciones térmicas o ambientales se perciben como aspectos de importancia secundaria y se reducen a aspectos de asoleamiento y calefacción. Si bien los residentes declaran apreciar la recepción directa de sol en algunas habitaciones, ello no necesariamente redunda en su utilización explícita, aunque sí se advierte en la funcionalización de los diferentes recintos, su iluminación y climatización. Asimismo, se reconoce una diferenciación térmica entre los niveles (por lo general más confortable el segundo nivel, aunque variable en el verano), la cual se asocia, no obstante, a una asumida mayor privacidad de los recintos de dormitorio que permitirían disfrutar aquella idoneidad.

En este mismo sentido se aplica el gasto en climatización, delegados sobre todo a equipos 
centrales de calefacción (en su mayoría a leña), y cuya gestión se organiza y distribuye entre el conjunto de los integrantes de la familia. Aquí se observa que ellos están orientados a otorgar buenas condiciones ambientales a los menores de edad, aunque también se muestran limitados para climatizar en términos ideales (debido a sus costos), lo que lleva al uso de pequeños calefactores móviles (eléctricos o a gas), en algunos recintos y periodos. La dimensión, materialidad o localización de los recintos no es reconocida por los residentes. Se utilizan escasamente las habitaciones pequeñas, menos iluminadas o más operativas (como las cocinas), pero también se valoran. Se aprecia el encuentro colectivo, pero se reconoce una vivencia crecientemente individualizada, con expectativas de una mayor permanencia personal, y controlada en los aspectos colectivos, en ámbitos cómodos y en relación con el exterior.

En las simulaciones, la mayor demanda energética es por calefacción en invierno (mayo-octubre), con bajos requerimientos de climatización en verano. Las necesidades de iluminación artificial, equipos domésticos y agua caliente sanitaria, permanecen más estables durante todo el año, con magnitudes aproximadas a los $300 \mathrm{~kW}$ h mensuales por vivienda, equivalentes a los registrados en los casos, mientras las demandas de calefacción varían de 6.000 a 18.000 kWh anuales, lo que dividido por la unidad de superficie corresponde de

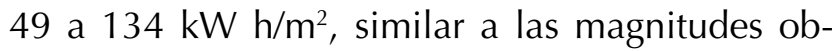
tenidas en otros análisis (Ambiente Consultores, 2007; Bustamante, 2009; CDT, 2010), aunque utilizando otros modelos, consideraciones y programas. Las demandas varían por el mayor tamaño de las viviendas como por sus condiciones constructivas. Estas magnitudes son similares a los consumos reales medidos en las viviendas por conceptos de calefacción, en gastos anuales de electricidad, gas, parafina o leña según la eficiencia estimada y poder calorífico (tabla 1), lo que permite reconocer que las simulaciones se aproximan a las situaciones reales al utilizar los parámetros establecidos para los modelos.
Al revisar los resultados por zonas térmicas (recintos) se advierten diferencias de demanda energética y condiciones ambientales. Ponderadas por la superficie, los valores de las distintas habitaciones en cada vivienda varían desde $12 \mathrm{~kW}$ h/ $\mathrm{m}^{2}$ hasta $330 \mathrm{~kW} \mathrm{~h} / \mathrm{m}^{2}$, en especial en las viviendas de mayor superficie construida (E y F). Estas diferencias se atribuyen a los diferentes estándares de calidad de las construcciones y la diversidad de los programas arquitectónicos. Es decir, según se observa en las simulaciones, se aprecian sectores de las viviendas con un desempeño natural adecuado durante todo el año (que prácticamente no requieren calefacción), mientras otros recintos presentan condiciones que demandan un alto consumo energético para alcanzar el rango de confort térmico definido.

El proceso de monitoreo evidencia que se alcanzan condiciones de confort solamente en periodos parciales del día, más extensos en verano, y de menor duración en invierno, con algunas mediciones específicas fuera de los rangos recomendables, por ejemplo en concentración de $\mathrm{CO}_{2}$ (sobre 1000 ppm) e iluminación. En el segundo nivel de las viviendas las temperaturas superficiales interiores presentaron una oscilación más moderada, ya que sus paramentos poseen menor transmitancia térmica. Las temperaturas del aire interior presentaron valores superiores por influencia de la ocupación y equipamiento, así como por estabilidad térmica, con una variabilidad similar pero con diferencias de hasta $5{ }^{\circ} \mathrm{C}$ entre recintos, evidenciando además desfases temporales. La humedad relativa presentó una menor oscilación respecto al exterior, pero también en magnitudes distintas entre habitaciones (en especial la cocina).

Las mediciones de flujometría confirmaron valores de transmitancia térmica consideradas en las simulaciones, lo que reconoce una adecuada ejecución de los elementos constructivos. Las termografías presentaron condiciones regulares de los paramentos, con algunos puentes térmicos en singularidades típicas de la construcción liviana asociada a los segundos pisos, o ampliaciones donde 
la estructura acusa la falta de material aislante o su discontinuidad. Las presurizaciones de aire presentaron un amplio rango de cambios de aire por hora (de 6 a $12 \mathrm{ACH}$ a 50 Pascales), pero consistentes con registros nacionales (Figueroa et al., 2013).

Las condiciones monitoreadas expresan situaciones habitacionales regulares, con carencias fundamentalmente en confort térmico de invierno (a pesar de la utilización de calefacción) y con altas infiltraciones de aire. Pero más aún, se reconocen diferencias en las mediciones por recintos, de acuerdo con su orientación solar, con incrementos de iluminación, temperatura y descensos de humedad, pero también variaciones producto de distintas condiciones de masa de la envolvente.

A continuación se analizan desempeños ambientales de recintos de distintas características arquitectónicas a partir de estos aspectos y basándonos en los tres criterios analíticos ya mencionados:

\section{Superficie expuesta}

Las simulaciones presentan diferencias similares de demanda por recintos, en que emerge como factor determinante la cantidad de superficie vertical (muros) expuesta al exterior. Las habitaciones con menos de $10 \mathrm{~m}^{2}$ al exterior poseen una media, en todos los casos analizados, de $116 \mathrm{~kW}$ $\mathrm{h} / \mathrm{m}^{2}$ de demanda anual para calefacción, mientras las habitaciones con más de $15 \mathrm{~m}^{2}$ expues-

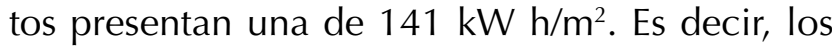
recintos localizados al centro de cada vivienda (con pocas caras hacia el exterior) requieren casi la mitad de energía para permanecer dentro del rango de confort en comparación con las habitaciones dispuestas en las esquinas expuestas de las viviendas. Esto se origina por la alta transmitancia térmica de la envolvente, que puede pensarse como posible de mitigar con mayor aislación en los muros, además de volúmenes más compactos, tal como ya han sugerido otras investigaciones (Bustamante, 2009; CDT, 2010). En la misma línea, las simulaciones indican que la demanda es mayor en las viviendas más irregulares y extendidas $(A, D, E)$, al generar habitaciones con mayor superficie expuesta.

En los monitoreos se efectuaron mediciones en cuatro recintos por vivienda en periodos limitados (una semana), por lo que es difícil proyectar desempeños generales. Sin embargo en algunos recintos con mayor exposición al exterior (dos o tres caras) se registraron temperaturas más bajas y más oscilantes, como también mayores porcentajes de humedad y $\mathrm{CO}_{2}$, (presumiblemente por mayor ocupación), así como de iluminación natural (ya que poseen ventanas mayores).

Según las observaciones, los residentes no valoran como aspecto determinante la magnitud de exposición de los recintos, ni tampoco que presenten un desempeño deficiente. Al contrario, la mayor disposición de ventanas que aportan mayor iluminación natural es un atributo evaluado positivamente, aunque ello esté en entredicho con la incidencia de menos en términos de temperatura y oscilación térmica que derivaría de la mayor superficie expuesta al exterior. Esta valoración es persistente como apreciación global, aunque no necesariamente incidente en una mayor o menor ocupación de los recintos.

\section{Orientación}

La posición de los recintos para recibir sol es la segunda diferencia más notoria que se desprende del análisis de las simulaciones. Las habitaciones con una cara al norte presentan una demanda anual

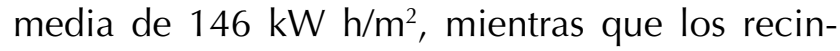
tos con caras al este/oeste o sur presentan $171 \mathrm{~kW}$ $\mathrm{h} / \mathrm{m}^{2}$, debido a la ganancia térmica por ventanas, como también en muros opacos. No obstante, hay una diferencia escasa o inversa en algunas viviendas ( $\mathrm{A}$ y $\mathrm{B}$ ) que poseen más frentes soleados.

De los monitoreos, se desprende que los recintos al sur presentan temperaturas más bajas, mientras que las de otros, al norte, son más altas, aunque también muestran una mayor oscilación térmica. 
Los ocupantes reconocen el asoleamiento como un factor relevante, asociado a una sensación de bienestar y calidez, aunque se asume que es un elemento no proyectable al conjunto de la vivienda ni posible de controlar en su totalidad.

\section{Localización vertical}

Los recintos ubicados en el primer nivel presentan una demanda anual media más alta $\left(161 \mathrm{~kW} \mathrm{h/ \textrm {m } ^ { 2 } )}\right.$ que los localizados en el segundo nivel o superior

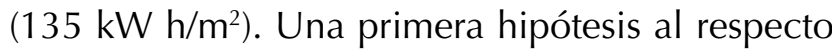
sugiere que se debe tanto a la mayor transmitancia térmica de la envolvente en el piso inferior, como al flujo térmico generado por los espacios verticales de comunicación (escaleras). De hecho, casi como reafirmando tal sugerencia, los casos $\mathrm{B}$ y $\mathrm{F}$ que poseen en el segundo piso paramentos con mayor transmitancia (menos aislación térmica) y escaleras más reducidas, tienen demandas similares o invertidas entre ambos niveles. Pero los datos obtenidos aún no permiten proyectar la hipótesis como concluyente, al no contarse con un número mayor de registros comparativos con los que contrastar la información.

De los monitoreos se advierte que los recintos inferiores presentan desempeños más bajos, mientras que los superiores registran un mejor comportamiento, si bien éstas presentan mayor humedad, $\mathrm{CO}_{2}$ e iluminación por su utilización.

Los residentes reconocen un mejor comportamiento térmico en los niveles superiores, tanto en sus expresiones verbales, como en el registro de mayor frecuencia de uso. Sin embargo, también reconocen el aporte simbólico y funcional de las habitaciones ubicadas en niveles inferiores, y muestran patrones de utilización más específicos y delimitados.

\section{Tamaño}

Los recintos con menor superficie horizontal (menos de $8 \mathrm{~m}^{2}$ ) presentan en las simulaciones una

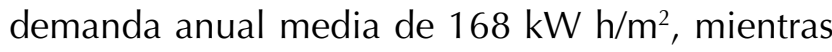

que las habitaciones mayores presentan un com-

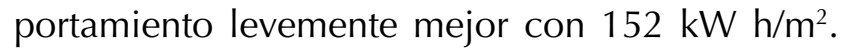
Esta diferencia puede originarse por la mayor relación de área envolvente, lo que incrementaría las transferencias internas de calor por oscilaciones climáticas. En las viviendas pequeñas (A y B), estas diferencias se reducen debido a la similitud de tamaño en los recintos.

En los monitoreos físicos no se midieron habitaciones menores dado que se buscaron recintos representativos y comparables entre los diferentes casos.

En los ocupantes se reconoce una apreciación inversa, ya que se asocia un desempeño más bajo a las habitaciones grandes, lo que es atribuible al tiempo requerido para calefaccionar $y$, por ende, para alcanzar el rango de confort.

\section{Función}

Las habitaciones que alojan el mismo uso (comedor, estar, cocina, dormitorios, baños) presentan diferencias similares de demanda según las simulaciones. El mejor desempeño se da en espacios

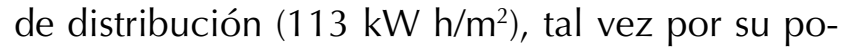
sición central; y el peor en las cajas de escalera (322 kW h/m ${ }^{2}$ ), eventualmente por su mayor relación de área envolvente, pero esto debe revisarse de manera más detallada. A su vez, los baños y dormitorios presentan mejor comportamiento (136

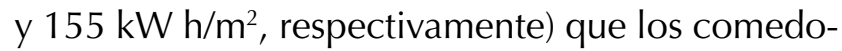

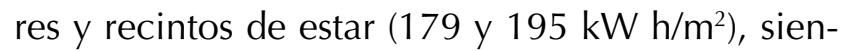

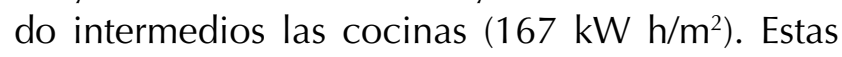
diferencias están influenciadas por su dimensión y localización interna. Las viviendas más grandes (D, E y F) tienen mayores diferencias entre habitaciones de distinto uso dado por la variedad de configuración de los recintos.

En los monitoreos se advierten diferencias similares, pero en la percepción de los residentes se reconocen desempeños inferiores en los baños, cocinas y circulaciones. Por otro lado, los dormitorios y recintos de estar se reconocen como de mejor comportamiento, debido, tal vez, a la 
utilización de equipos de calefacción, ya que estos están en funcionamiento permanente.

\section{CONCLUSIONES}

Este trabajo expone una revisión del desempeño ambiental de viviendas de una misma zona climática, analizadas a partir de tres fuentes de recopilación de datos, diferenciados por recintos. Aunque pueden advertirse limitaciones en los procesos de medición, es posible reconocer características de las habitaciones que inciden en el confort interior.

En términos generales, las simulaciones indican demandas térmicas con resultados muy distintos por recintos, parcialmente consistentes entre casos y con algunas características comunes a tener en cuenta. Por su parte, las monitorizaciones entregan valores ambientales diferentes entre algunas habitaciones, pero por periodos breves. Por último, las entrevistas y observación de ocupación de los residentes expresan percepciones variables por sectores que son cercanas, o medianamente coherentes, con las simulaciones y monitoreos. De acuerdo con estos resultados, se puede afirmar que el estudio identifica condiciones arquitectónicas de recintos, que afectan el desempeño ambiental y consumo energético, que deben considerarse en los nuevos diseños y rehabilitaciones de viviendas existentes para asegurar una mejor calidad residencial.

Respecto a las características de las habitaciones, se desprende de las simulaciones que los recintos con menor cantidad de superficie expuesta al exterior presentan una demanda térmica más baja. Por tanto, siguiendo esta pauta, los proyectos de viviendas en una zona climática similar podrían considerar una área reducida de envolvente (o sea, volúmenes compactos), y ubicar las habitaciones más utilizadas en la parte central de la casa. En la modificación de construcciones existentes se deberían examinar ampliaciones que completen las formas (para evitar aumentar la superficie total expuesta) y privilegiar el mejoramiento térmico de las envolventes perimetrales, promoviendo así los frentes y habitaciones hacia el costado más soleado, que fue la segunda característica que otorgó menor demanda térmica. Y que también es reconocida en las monitorizaciones y apreciada por los residentes.

Así mismo, se advierte cruzando la información recopilada que existe una menor demanda y mayor valoración de los niveles superiores de las viviendas, junto con la preferencia por habitaciones con mayor luminosidad. Para promover estas condiciones en el diseño o rehabilitación de viviendas la opción pasaría por extender el volumen, aunque ello significaría contravenir la compacidad recomendada a partir de las simulaciones. Una alternativa apuntaría a considerar ajustes particulares, desarrollando algunos recintos con mayor relación al exterior y envolvente de baja transmitancia.

En el caso del tamaño y función de los recintos, advertimos que se relacionan levemente con la demanda térmica y dependen de la organización espacial de las viviendas, por tanto no sugieren ajustes relevantes. Sin embargo, parece recomendable separar sectores de ocupación más ocasionales, incrementando la aislación perimetral y promoviendo iluminación natural para la habitabilidad.

Completando esta reflexión se puede considerar que las diferencias entre recintos en las viviendas revelan fundamentalmente un desempeño heterogéneo de la habitabilidad doméstica. La envolvente general modera las inclemencias climáticas, pero se advierten variaciones interiores que requieren esfuerzos en calefacción central, y en algunos periodos, aportes especiales en algunos recintos expuestos. Esto expresa una situación de confort diferenciado, con condiciones básicas para toda la vivienda, pero con lugares privilegiados que se relacionan con el exterior. Esta diversidad ya ha sido sugerida en otras investigaciones recientes (Frontzack, 2011), y deberían considerarse en los estudios residenciales.

Teniendo en cuenta los hallazgos de este estudio, se recomienda realizar análisis más exhaustivos y comparativos entre los distintos recintos 
que componen las viviendas. El tratamiento más especializado de este aspecto indica que se pueden promover importantes mejoras en el confort doméstico, además de una atenuación sustancial del impacto energético, lo que permite a su vez una disminución de los gastos domésticos. En este sentido, una mayor profundización de lo realizado, con una mayor cantidad de casos para analizar, permitiría fomentar un desarrollo residencial con mejor desempeño ambiental y habitabilidad. Porque, en definitiva, esta investigación muestra que de la revisión detallada de los supuestos de confort ambiental residencial (analizados en las diferenciaciones espaciales y temporales de recintos y formas de ocupación), surgen aspectos que son importantes para determinar acciones de mayor impacto sobre la calidad interior residencial y demanda energética asociada.

\section{FINANCIAMIENTO}

Este trabajo ha recibido financiamiento del proyecto de investigación Fondecyt 1120165 del Gobierno de Chile, y se agradece la participación de colaboradores y residentes de las viviendas analizadas.

\section{REFERENCIAS}

Ambiente Consultores (2007). Programa de inversión pública para fomentar el reacondicionamiento térmico del parque construido de viviendas. Santiago: Ministerio de Vivienda y Urbanismo.

Anderson, K. (2014). Design Energy Simulation for Architects. Nueva York: Routledge.

Beizaee, A.; Lomas, K. \& Firt, S. (2013). National Survey of Summertime Temperatures and Overheating Risk in English Homes. Building and Environment 65(1), 1-17.

Bustamante, W. (2009). Guía de diseño para la eficiencia energética de la vivienda social. Santiago: Ministerio de Vivienda y Urbanismo.

Cámara Chilena de la Construcción (2011). Balance de la Vivienda en Chile. Santiago.
Centro de Desarrollo Tecnológico (CDT) (2010). Estudio de usos finales y curva de oferta de conservación de la energía en el sector residencial. Estudio final y resumen ejecutivo. Santiago: Cámara Chilena de la Construcción.

Chiou, Y. (2009). An Ocupant-Driven Integrative Household System Form Residential Building Energy Simulation. Recuperado el 20 abril de 2014, de: http://www.cmu.edu: http://www.cmu.edu/architecture/research/grad_work/2009_phdbpd_chiou_ yunshang.pdf

Elzeyadi, I. (22-24 de junio de 2009). Architectural Pride and Environmental Prejudice: The Effect of Personal Status, Historical Value, and Indoor Décor on Occupants Indoor Environmental Quality in Offices. Recuperado el 21 de junio de 2013, de: www. plea2009.arc.ulaval.ca: www.plea2009.arc.ulaval. ca/attaches/awards/2-3-01-PLEA2009Quebec.pdf

Fanger, P. (1973). Assessment of man's thermal comfort practice. British Journal of Industrial Medicine, 30, 313-324.

Figueroa, R.; Bobadilla, A.; Besser, D.; Díaz, M.; Arriagada, R. y Espinoza, R. (12 de septiembre de 2013). Air Infiltration in Chilean Housing: A Baseline Determination. Recuperado el 12 de junio de 2014, de: mediatum.ub.tum.de: mediatum.ub.tum.de/ doc/1169604/1169604.pdf

Fissore, A. (2012). La realidad energética en el sector residencial de la Región del Bío Bío. Santiago: EPCAmericas.

Frontczak, M. (2011). Human comfort and self-estimated performance in relation to indoor environmental parameters and building features. Tesis Doctoral, DTU Civil Engineering Report R-260. Reino Unido. Gómez-Aspeitía, G.; Bojórquez, G. y Ruiz, R. (2007). El confort térmico: dos enfoques teóricos enfrentados. Palapa 2(1), 45-57.

Hendron, R. \& Engebretch, C. (2010). Building America House Simulation Protocols. Tennessee: National Renewable Energy Laboratory.

Hensen, J. \& Lambert, R. (2011). Building Performance Simulation for Design and Operation. Londres: Routledge. 
Jankovic, L. (2012). Designing Zero Carbon Buildings Using Dynamics Simulation Methods. Nueva York: Routledge.

Jirón, P. (2011). On Becoming 'La Sombra/The Shadow'. En: M. Buscher, J. Urry \& K. Witchger. Mobile methods (pp. 36-53). Nueva York: Routledge.

Jirón, P. e Iturra, L. (2011). Momentos móviles. Los lugares móviles y la nueva construcción del espacio público. Arquitecturas del Sur, (39), 44-57.

Krygiel, E. \& Nies, B. (2008). Green BIM: Succesful Sustainable Design with Building Infomation Modeling. Indianapolis: Wiley.

León, L.; Muñoz, S.; León, J. y Bustamante, P. (2011). Monitorización de variables medioambientales y energéticas en la construcción de viviendas protegidas: edificio Cros-Protecnia en Sevilla. Informes de la construcción 62(519), 67-82.

Peeters, L.; Dear, R.; Hensen, J. \& D'Haeseleer, W. (2009). Thermal Comfort in Residential Buildings: Comfort Values and Scales for Buildings Energy Simulation. Applied Energy 86(5), 772-780.

Sarmiento, P. y Hormazábal, N. (2003). Habitabilidad térmica en las viviendas básicas de la zona central de Chile a la luz de los resultados preliminares del Proyecto Fondef D00I103941. Revista INVI 46(18), 23-32.

Smith, V. (2012). Modeling Building Thermal Response to HVAC Zoning. ACM SIGBED Review 9(3), 39-45. 